\title{
Primary Gallbladder Adenocarcinoma in a Cat
}

\author{
Taiani Torquato Diogenes ${ }^{1}$, Mariana Araújo Rocha ${ }^{2}$, Keytyanne de Oliveira Sampaio ${ }^{3}$, Robério Gomes Olinda ${ }^{4}$, \\ Diana Celia Sousa Nunes Pinheiro ${ }^{5} \&$ Reginaldo Pereira de Sousa-Filho ${ }^{5}$
}

\begin{abstract}
Background: Biliary tumors have low incidence in cats and are more common in elderly animals. Hepatobiliary tumors have different classifications and their etiology is difficult to interpret. In most cases, the diagnosis is only possible in advanced stages, which clouds the precursor factors. The late diagnosis is explained by the absence or specificity of clinical manifestations and laboratory changes. The majority of hepatobiliary neoplasms in cats are incidental findings in surgeries or necropsies. This study aimed to report clinical, laboratory, pathological and immunohistochemical results in a feline case of gallbladder adenocarcinoma.

Case: 4-year-old male castrated mix breed cat was admitted at a veterinary clinic with a history of polyuria / polydipsia, anorexia, apathy, jaundice and emesis for 60 days. The animal had been treated in another clinic with silymarin, famotidine and cyanocobalamin, and fed by esophageal tube based on the presumptive clinical diagnosis of cholangitis. The clinical examination revealed jaundice, abdominal pain, weight loss, enlargement of the liver and gallbladder and the presence of a structure in the epigastric region. Based on clinical signs, blood tests (complete blood count and liver enzymes), abdominal ultrasound and thoracic radiography were requested. In the exam results, eosinophilia, bilirubinemia and increased alkaline phosphatase, glutamic pyruvic transaminase and gamma glutamyl transferase were observed, in addition to the presence of icteric and hemolyzed serum. Ultrasound exam revealed thickened and dilated cystic and common ducts, large and thick gallbladder, bile with bile mud, hypoechogenic liver, thickening in the duodenal papilla and enlarged pancreatic and duodenal lymph nodes. There were no alterations in thoracic radiography. Based on the findings, the diagnosis of extrahepatic biliary obstruction was suggested. In celiotomy, dilated gallbladder with a thick blackened wall was observed. Bile puncture was performed for analysis, and total cholecystectomy and removal of obstructive content was performed. The patient suffered cardiorespiratory arrest after the procedure. A fragment of gallbladder and liver were sent to evaluation with histopathology and immunohistochemistry, and results were compatible with gallbladder adenocarcinoma.

Discussion: Hepatobiliary neoplasms in cats are uncommon, apart from lymphoma. The incidence is higher in elderly cats and in males, which did not match the animal in this case. The clinical presentation of the condition is quite nonspecific, corroborating with other reports, in which changes are often only evident in advanced stages of the disease. Laboratory and imaging findings pointed to a biliary tree disorder, and cholangitis was suggested. However, the lack of clinical improvement and examination findings of exams lead to an exploratory laparotomy for better inspection of the organs and identification of possible causes. One of the differential diagnoses that should be considered in cats with extrahepatic biliary obstruction is gallbladder adenocarcinoma, especially in animals with an unsatisfactory response in clinical treatment. Tests such as histopathology and immunohistochemistry are essential for the definitive diagnosis of this neoplasm. Surgical resection is indicated in cases of malignant masses, providing longevity and better quality of life. Hypotension is a common complication in hepatobiliary surgeries, which can result in death.
\end{abstract}

Keywords: feline, immunohistochemical, liver, neoplasm, surgery.

${ }^{1}$ Equalis, Programa de Pós-Graduação, Natal, RN, Brazil. ${ }^{2}$ Hospital Veterinário Jerônimo Dix-Suit Rosado Maia, Universidade Federal Rural do SemiÁrido (UFERSA), Mossoró, RN. ${ }^{3}$ Departamento de Medicina Veterinária, Universidade Federal Rural de Pernambuco (UFRPE), Recife, PE, Brazil. ${ }^{4}$ Centro de Ciências da Saúde, Universidade de Fortaleza (UNIFOR), Fortaleza, CE, Brazil. ${ }^{5}$ Faculdade de Medicina Veterinária, Universidade Estadual do Ceará (UECE), Fortaleza. CORRESPONDENCE: T.T. Diogenes [taianitorquato@ gmail.com] \& M.A. Rocha [mariana4981@hotmail.com].Faculdade de Medicina Veterinária, UECE. Av. Dr. Silas Munguba n. 1700. Campus Itaperi. CEP 60.740-903 Fortaleza, CE, Brazil. 


\section{INTRODUCTION}

Primary gallbladder neoplasms are rare in cats, in which incidence values vary from 1 to $3 \%$ among different types [11]. These conditions are more common in elderly animals and breed-related predispositions have not been reported [1].

Hepatobiliary neoplasms are classified as hepatocellular adenomas, hepatocellular adenocarcinomas, cholangiocellular adenomas, cholangiocellular adenocarcinomas or mesenchymal tumors. The cholangiocellular adenocarcinomas include intrahepatic bile duct adenocarcinomas, extrahepatic bile duct adenocarcinomas and gallbladder carcinomas [3].

The etiologies of gallbladder tumors are hard to interpret. In most cases, diagnosis is confirmed only in advance stages of the disease, which hinders the identification of precursor lesions [7]. Late diagnosis may be explained by the clinical presentation that is absent in $50 \%$ of cases and is composed by nonspecific signs, such as inappetence, weight loss, lethargy and emesis [6].

Laboratory alterations, such as the increased levels of serum liver enzymes are also unspecific [1]. Hence, half the cases of hepatobiliary tumors in cats are incidental findings of surgeries or necropsies [4].

This study aimed to report clinical, laboratorial, pathological and immunohistochemical findings in a case of gallbladder adenocarcinoma in a cat.

\section{CASE}

A 4-year-old male castrated mix breed cat was admitted at a veterinary clinic for consultation. According to the owner, the individual presented a history of apathy, polyuria/polydipsia, anorexia, emesis and jaundice for 60 days. Based on a previous diagnosis of cholangitis in another clinic, the individual was being treated orally for 40 days with Silymarin ${ }^{1}[40 \mathrm{mg} / \mathrm{kg}$, twice a day BID], Famotidine ${ }^{2}[1 \mathrm{mg} / \mathrm{kg}$, once a day - SID], and with Cyanocobalamin ${ }^{3}$ [250 $\mu$ g, via subcutaneous injections every 7 days]. Patient was being fed through an esophageal tube with hypercaloric pasty feed for 30 days. In the clinical examination, emaciation was observed with a body score of 4 (1-9 scale). In addition, the individual presented jaundice in skin and mucosa, hepatomegaly, abdominal pain and a mass was identified in the epigastric region. In view of the situation, some tests were requested, such as complete blood count, biochemical enzyme activity (direct-bilirubin, free-bilirubin, indirect-bilirubin, Alkaline
Phosphatase - AP, Glutamic Pyruvic Transaminase - GPT and Gamma-Glutamyl Transferase - GGT), total abdominal ultrasound and thoracic radiography.

Leukogram results demonstrated intense eosinophilia (2.624 cells/ $\mu \mathrm{L}$ - reference: $0-1,500$ cells/ $\mu \mathrm{L} / \mathrm{L})$. Serum biochemical profile presented elevated levels of bilirubin (Direct bilirubin $11.4 \mathrm{mg} / \mathrm{dL}$ - reference: $0.30 \mathrm{mg} / \mathrm{dL}$; Indirect bilirubin $4.6 \mathrm{mg} / \mathrm{dL}$ - reference: 0.0-0.5 mg/dL) liver enzymes (FA 255 $\mathrm{mg} / \mathrm{dL}$ - reference: $25.0-93.0 \mathrm{mg} / \mathrm{dL}$; TGP $527 \mathrm{mg} / \mathrm{dL}$ - reference: 6.0-83.00 mg/dL; GGT $40.00 \mathrm{mg} / \mathrm{dL}$ - reference: $1.3-5.10 \mathrm{mg} / \mathrm{dL}$ ), icteric and hemolyzed serum.

In abdominal ultrasonography, the liver presented reduced and gross echogenicity with no evidence of nodules or cysts. Gallbladder presented severely thick echogenic walls $(0.35 \mathrm{~cm})$, anechoic bile with hypoechoic content in the lowest part (biliary mud). Cystic and common ducts were dilated $(0.46 \mathrm{~cm}$ and $0.29 \mathrm{~cm}$ in diameter, respectively) and thick $(0.09 \mathrm{~cm}$ in both). Duodenum was also thick in the duodenal papilla $(0.30 \mathrm{~cm})$ and pancreatic parenchyma was slightly hypoechoic. Lymphadenomegaly was observed in pancreatic and duodenal lymph nodes. Thoracic radiography did not present alterations.

Considering the case and the unsatisfactory therapeutic response, a possible diagnosis of extrahepatic biliary obstruction was considered. Hence, a surgical approach was suggested to perform a bilioenteric bypass. Maintenance fluid therapy was recommended, and pain was managed using Meloxicam ${ }^{4}$ [0.05 mg/kg, SC, SID] for 3 days. The animal was submitted to surgery, in which pre-anesthesia consisted of Methadone ${ }^{5}[0.3 \mathrm{mg} /$ $\mathrm{kg}, \mathrm{SC}]$ and Dexmedetomidine ${ }^{6}(3 \mu / \mathrm{kg}, \mathrm{SC})$. Isoflurane was used for induction via mask and in maintenance at $2 \%$. In the celiotomy, gallbladder was severely dilated (Figure 1) and manual decompression was not possible. In addition, the left liver duct, cystic duct and common bile duct were ingurgitated. Gallbladder walls were thick and dark. Hyperemia was observed in proximal duodenal walls, omentum and peripancreatic fat, in addition to lymphadenomegaly. Based on these findings, total cholecystectomy was performed. At the end of surgery, a severe hypotension occurred, which resulted in cardiorespiratory arrest. Resuscitation protocol was performed with no success. A bile sample was collected for cytology and bacterial culture. The excised gallbladder and a liver fragment were sent for histopathological analysis. Bacterial culture was negative and cytology 
results demonstrated squamous epithelial cells, neutrophils, macrophages and amorphous acellular material with no evidence of bacteria or parasite eggs.

In histopathological examination, marked proliferation of unencapsulated carcinoma epithelial cells which infiltrated the mucosa and submucosa of the gallbladder was observed. Neoplastic cells formed irregular acini and papillary structures, which were sustained by abundant fibrovascular stroma (marked desmoplasia) [Figure 2A] . Multifocal intramural inflammatory infiltrate of neutrophils and eosinophils was observed. Tumoral clots were seen in lymph and blood vessels, in addition to necrotizing vasculitis and fibrin thrombi in submucosal vessels. In the liver fragment adjacent to the gallbladder, discrete fibrosis and marked proliferation of periportal biliary ducts was observed. In addition, a fine diffuse hepatocellular vacuolization was identified.

According to the histopathological findings, gallbladder adenocarcinoma was diagnosed. Samples from these tissues were submitted to immunohistochemical analysis, in which four biomarkers were used in the peroxidase streptavidin-biotin technique with the monoclonal antibodies Anti-Hepatocyte Specific Antigen (Clone OCH1E5) ${ }^{7}$, Anti-CK Pan (Clone AE1AE3) ${ }^{8}$, Anti-CK7 (Clone OV-TL12/30) ${ }^{9}$ and CK20 (Clone Ks20.8) ${ }^{9}$. Immune expressions for CK Pan and CK7 were observed, which confirmed the diagnosis (Figure 2B,D) [Figure 2C].

\section{DISCUSSION}

Hepatobiliary neoplasia in cats is relatively uncommon with the exception of lymphomas [9]. A study conducted by Patnaik [10], demonstrated that among hepatobiliary tumors in cats, those of epithelial origin and biliary system were most frequent, unlike humans and dogs, in which liver neoplasia are more common.

Primary hepatobiliary tumors occur more frequently in older and male individuals with average age between nine and ten years [12]. However, the animal in this case was much younger (4-year-old).

Clinical presentation presented by the cat in this report consisted of apathy, anorexia and emesis, which are unspecific. This finding corroborates with [9] that reported these signs in hepatobiliary cases. According to Liptak et al. [6], only $50 \%$ of hepatobiliary neoplasia cases in cats present clinical manifestations, which are nonspecific and mostly identified in advan- ced stages of the disease. Other clinical manifestations include weight loss, jaundice, hepatomegaly, polyuria and polydipsia $[4,12]$.

The main biochemical alterations that were identified in this case are unspecific for the tumor and could occur in several conditions affecting liver and biliary tree, including increased liver transaminases and bilirubin [9]. Other expected laboratory findings include neutrophilia and chronic anemia, which were not observed in this report [1].

Clinical and laboratory alterations suggested the initial diagnosis of cholangitis. However, the lack of improvement after the clinical treatment and the ultrasonographic findings suggestive of obstructive condition, especially due to the dilation of common and cystic ducts led to the extrahepatic biliary duct obstruction diagnosis [2]. In addition, the presence of biliary mud suggests cholestasis, which is considered a significant finding in cats [4].

This condition may occur as a consequence of chronic cholangitis, pancreatitis, gallstones, parasite infestation and neoplasms [9]. Hence, all of these conditions are considered as differential diagnoses of gallbladder adenocarcinoma. Surgical exploration for biliary decompression and inspection of liver and other organs is recommended when the cause is not identified or in the absence of clinical improvement after the treatment is initiated [2], which was the procedure adopted in this case.

Neoplasm was demonstrated by the malignant epithelial cell infiltrate in mucosa and submucosa of the gallbladder. These cells presented immune expression by CK7, which marks ductal, glandular, superficial squamous and transition epitheliums, and by CK Pan, which is a specific antigen marker of filaments of epithelial cells that characterizes adenocarcinoma [5].

Surgical resection is indicated to provide better duration and quality of life for the animal [12]. Chemotherapy does not present adequate results in the treatment of primary hepatobiliary tumors [1]. During surgery, the occurrence of hypotension is common in cat anesthesia during procedures involving gallbladder and biliary tree, which can result in death [8] as occurred in this case. A mechanism that may affect muscle tonus in these patients is the presence of increased bilirubin concentrations in blood. In addition, a possible increased liberation of nitric oxide associated with obstructive jaundice have been reported in dogs [8]. 
Prognosis for malignant hepatobiliary neoplasia is unfavorable with elevated metastasis rates [3], which were not observed in this case. However, the

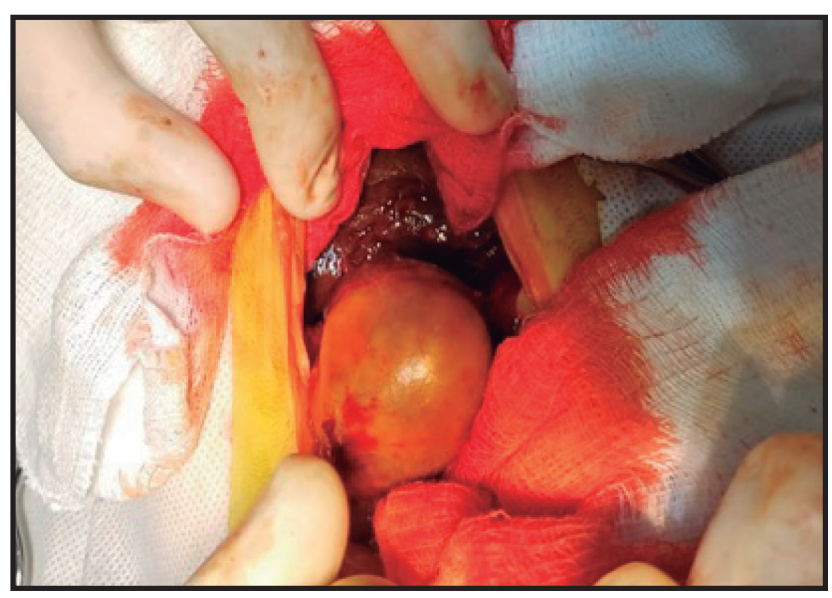

Figure 1. Gallbladder carcinoma in a cat. Marked dilation and thickening of gallbladder walls. possibility was clear, considering the tumoral clots identified in histopathological analysis.

The gallbladder adenocarcinoma should be considered as a differential diagnosis for extrahepatic biliary obstruction in cats, especially in cases that are unresponsive to clinical treatment. Histopathology and immunohistochemistry are key tests to confirm the presence of this neoplasia. Despite the risk of death during surgery, the indicated treatment is surgical resection with the aim to promote better quality of life for the animal.

\section{MANUFACTURERS}

${ }^{1}$ Takeda Pharma Ltda. Jaguariúna, SP, Brazil.

${ }^{2}$ Aché Laboratórios Farmacêuticos S.A. Guarulhos, SP, Brazil.

${ }^{3}$ Laboratório Bravet Ltda. Rio de Janeiro, RJ, Brazil.

${ }^{4}$ Ouro Fino Saúde Animal Ltda. Cravinhos, SP, Brazil.

${ }^{5}$ Cristália Produtos Químicos Farmacêuticos Ltda. Itapira, SP, Brazil. ${ }^{6}$ Zoetis Inc. Parsippany, NJ, USA.

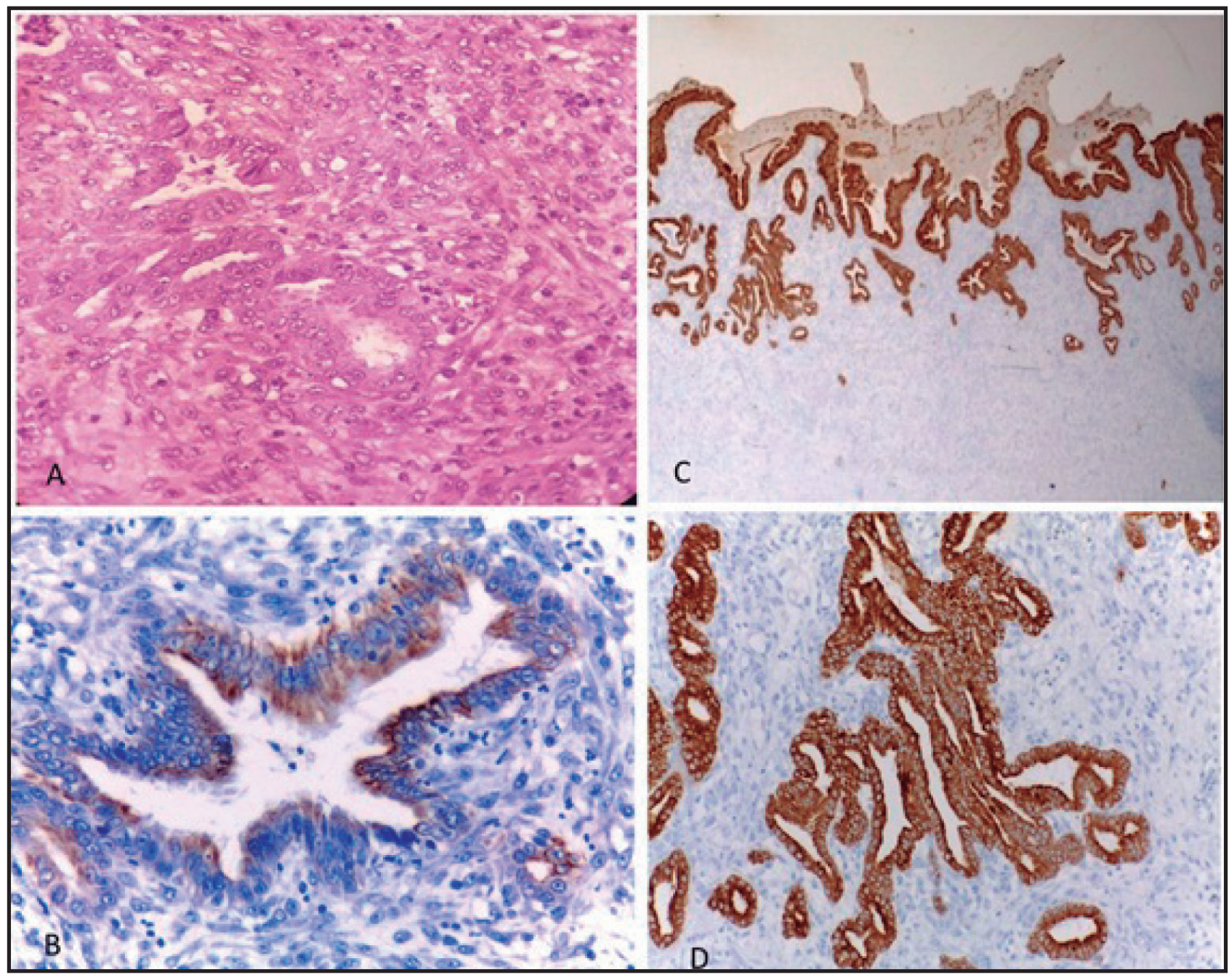

Figure 2. Gallbladder carcinoma in a cat. A- Marked proliferation of carcinoma cells with acini-like structures. Cell morphology range from polygonal to cuboidal with scarce to moderate eosinophilic cytoplasm. Nuclei are round to oval with vacuolated chromatin and evident nucleoli [H.E.; Obj. 20x]. B- Intense cytoplasmatic immune marking of epithelial cells for CK Pan [Obj. 10x]. C- Moderate cytoplasmatic immune marking of epithelial cells for CK7 [Obj. 40x]. D- Intense cytoplasmatic immune marking of epithelial cells for CK Pan [Obj. 20x]. 
${ }^{7}$ Abcam Plc. Cambridge, RU, UK.

${ }^{8}$ Thermo Fisher Scientific Inc. Carlsbad, CA, USA.

${ }^{9}$ Dako North America Inc. Carpinteria, CA, USA.
Declaration of interest. The authors report no conflicts of interest. The authors alone are responsible for the content and writing of paper.

\section{REFERENCES}

1 Balkman C. 2009. Hepatobiliary neoplasia in dogs and cats. Veterinary Clinics of North America: Small Animal Practice. 39(3): 617-625.

2 Center S.A. 2009. Diseases of the gallbladder and biliary tree. Veterinary Clinics of North America: Small Animal Practice. 39(3): 543-598.

3 Cullen J.M. 2017. Tumors of the Liver and Gallbladder. In: Meuten D.J. (Ed). Tumors of Domestic Animals. 5th edn. Ames: John Wiley \& Sons Inc., pp.602-631.

4 Harran N., D’Anjou M.A., Dunn M. \& Beauchamp G. 2011. Gallbladder sludge on ultrasound is predictive of increased liver enzymes and total bilirubin in cats. The Canadian Veterinary Journal. 52(9): 999-1003.

5 Hornick J.L., Lauwers G.Y. \& Odze R.D. 2005. Immunohistochemistry Can Help Distinguish Metastatic Pancreatic Adenocarcinomas from Bile Duct Adenomas and Hamartomas of the Liver. The American Journal of Surgical Pathology. 29(3): 381-389.

6 Liptaik J.M., Dernell W.S. \& Withrow S.J. 2004. Liver tumors in cats and dogs. Compendium on Continuing Education for the Practising Veterinarian. 26(9): 50-56.

7 Meyer A., Jukemura J. \& Penteado S. 2011. Tumores e pólipos de vesícula biliar. In: Zaterka S. \& Eisig J.M. (Eds). Tratado de Gastroenterologia da Graduação à Pós-Graduação. São Paulo: Atheneu, pp.877-882.

8 Monticelli P., Stathoupolou T.R., Lee K. \& Adami C. 2017. Life-threatening perianaesthetic complications in five cats undergoing biliary tract surgery: case series and literature review. Journal of Feline Medicine and Surgery. 19(6): 717-722.

9 Otte C.M., Penning L.C. \& Rothuizen J. 2017. Feline biliary tree and gallbladder disease: aetiology, diagnosis and treatment. Journal of Feline Medicine and Surgery. 19(5): 514-528.

10 Patnaik A.K. 1992. A morphologic and immunocytochemical study of hepatic neoplasms in cats. Veterinary $P a-$ thology. 29(5): 405-415.

11 Patnaik A.K., Lieberman P.H., Erlandson R.A. \& Antonescu C. 2005. Hepatobiliary neuroendocrine carcinoma in cats: a clinicopathologic, immunohistochemical, and ultrastructural study of 17 cases. Veterinary Pathology. 42(3): 331-337.

12 Wypj J., Fan T.M. \& Lorimier L.P. 2006. Primary hepatic and biliary tract tumors in dogs and cats: an overview. Veterinary Medicine. 101(6): 384-394. 